\title{
ANALITIC INVESTIGATION OF THE REGULARITIES OF CHANGING DUST CONCENTRATION DURING THE ABRASIVE DECREASE OF STONE STRUCTURES
}

\author{
Ala Bezpalova \\ Department of Building Management and Labor Protection \\ Odessa State Academy of Civil Engineering and Architecture \\ 4 Didriksona str., Odessa, Ukraine, 65029 \\ bespalova-a-v@mail.ru \\ Vladimir Lebedev \\ Department of Structural Materials Technology and Materials \\ Odessa National Polytechnic University \\ 1 Shevchenko ave., Odessa, Ukraine, 65044 \\ wlebedev29@rambler.ru \\ Yuri Morozov \\ Department of higher mathematics and systems simulation \\ Odessa National Polytechnic University \\ 1 Shevchenko ave., Odessa, Ukraine, 65044 \\ morozovyu@gmail.com
}

\begin{abstract}
In the process of repair or restoration of building structures, it is often necessary to strengthen building structures from limestone-shell rock, concrete, reinforced concrete, hard materials-granite, basalt, etc. by cutting or making cuts of the required size with detachable circles of synthetic diamond and cubic boron nitride (CA and $\mathrm{CBN}$ ).

The cutting process is accompanied by considerable dust formation, which can be both harmful and dangerous factor in the work.

The aim of the work is studying the process of dust sedimentation and the regularity of the change in dust concentration during the abrasive cutting of concrete and stone materials.

Mathematical models have been developed - dust emission from under the wheel, speed of sedimentation of dust particles depending on their material, size and shape, and also depending on temperature, pressure and humidity, the concentration of dust in the working space and the concentration change during the cutting cycle are calculated.

It is shown that the velocity of the sedimentation of particles depends significantly on the shape. The higher the sphericity, the higher the sedimentation rate. The ambient temperature has little effect on the sedimentation rate, in the temperature range $\left(-20 \rightarrow+40^{\circ} \mathrm{C}\right)$ at which the operation takes place.

The sedimentation rate of dust particles generated by cutting the most common building stone materials also differs slightly. Almost the same sedimentation rate has dust particles obtained by cutting basalt and concrete. A bit higher is the sedimentation rate of particles from granite.

The sedimentation rate of particles of generated dust is about $600-700 \mathrm{~cm} / \mathrm{h}$ or $10-11 \mathrm{~cm} / \mathrm{min}$ for particles measuring $6 \mu \mathrm{m}$. This means that at a production height of about $2 \mathrm{~m}(200 \mathrm{~cm})$ during the operating cycle (about $3 \mathrm{~min})$, the dust will remain at an altitude of about $1.5 \mathrm{~m}$, i. e. practically remains in the working area. This gives grounds to assert about a high concentration of dust during the cutting cycle (about $4.8108 / \mathrm{m}^{3}$ ).
\end{abstract}

Keywords: cutting of stone structures, dust formation, dust sedimentation, dust concentration, sedimentation rate, sedimentation time.

\section{Introduction}

In work [1] the mechanism of dust generation during abrasive cutting of stone structures was shown. A minute concentration of dust in the working space was shown. However, the concentration of dust is not exhausted by this, so let's consider this issue in more detail. In the process 
of repair and restoration of buildings, it is often necessary to strengthen structures that have great destruction. In this case, auxiliary structures are introduced into the walls, foundations and floors, for which they have to cut openings and nests, where reinforcing elements are built.

Such works are often performed in limestone-shell, concrete, granite, basalt by cutting or making cuts to the required size with detachable circles of synthetic diamond and cubic boron nitride (CA and $\mathrm{CBN}$ ). Cutting of solid building materials is carried out by disk diamond circles and circles from CBN rotation speed, which, and, consequently, the cutting speed is $35-50 \mathrm{~m} / \mathrm{s}$. In view of the high intensity of the cutting process and intensive micro-striking, the cutting process is accompanied by considerable dust formation, as indicated in [1]. The dust concentration in the working space can't be characterized by only a minute concentration. The cutting process is long, at least a few minutes. If divide the dust into minute portions, it is obvious that after the first portion has arrived, it is partially in the air for some time. When it is in the air, a second portion of dust enters the working space, followed by a third, and so on. Thus, the longer the dust sedimentation time, the higher its concentration in the working space.

The time of dust sedimentation depends on many factors - the height at which the cutting takes place (when cutting through the window openings this height can be about three meters), the size of the dust particles, the shape of the dust particles, the state of the environment, the dust particles.

It should be noted that when cutting metal objects, the separation of chips and dust occurs as a result of plastic shear deformation. In this case, the shape of the shavings - a speck of dust - is close to the shape of a "comma", which can be taken into account in practical calculations. However, when cutting a brittle material from which stone building structures are made, the formation of a dusty mote occurs as a result of brittle fracture, in which the shape of the dust particles is arbitrary, which naturally affects the sedimentation rate (sedimentation) of the dust particle.

Building structures can consist of dissimilar materials - different density and hardness. Dusts of different chemical composition, even with the same size and shape, will have different rates of sedimentation, and, consequently, will be deposited at different times.

It is not difficult to see that when cutting, dust from under the circle literally flies out along the tangent to the circle. The direction can be arbitrary depending on the position in which work is performed, including upwards, that will add the height of the dust ejection.

In addition, it is necessary to take into account the state of the environment - temperature and humidity, which will affect the density of this environment and the Archimedean forces that act on the dust particle.

All these factors should be taken into account, both in theoretical calculations and in experiments, if the latter are to be performed.

It is more reasonable to have mathematical models of dust ejection, sedimentation rate and sedimentation time to determine all these dependencies, depending on the above factors.

The existence of such models can make it possible to conduct a computational experiment, establish the necessary dependencies, determine the concentration of dust during working hours, which in turn will provide material for improving and, if necessary, developing new means of individual and collective protection of workers.

\section{Analysis of literary data and the formulation of the problem}

At present, there are theoretical models of dust sedimentation.

So work [5] has mathematical models of dust sedimentation, which take into account the various forces that act on the dust particle during its waking in the air. However, the calculations refer to dust emitted from chimneys of considerable height 40 to $50 \mathrm{~m}$. Thus, it is rather a matter of soot particles. The shape of the particles is not taken into account, it is known that the basic shape of the particles is flaky. The mineral composition of dust and the dependence of the speed of sedimentation on this composition are not considered.

In work [6] there are mathematical models of dust sedimentation in production shops of construction enterprises, however without sources of dust generation. The height with which the dust precipitates is not specifically indicated, however, the authors consider the shape of the dust 
grains, although the main calculations are made for spherical dust particles. One type of material is considered - mineral wool. Pathogenic properties of this type of dust are very high, however, dust of other mineral composition is not considered.

In work [2] there are models of dust sedimentation in ventilation ducts. The authors tried to take into account all types of forces that act on the speck of dust while it is in the air. Mathematical models basically take into account the spherical shape of the particles. The mineral composition of the dust is also not considered.

In work [3], the sedimentation velocities of particles from various materials - minerals, biomaterials, biomineral in water - are considered. The mathematical apparatus takes into account all the forces acting on the particle, but the environment in which the sedimentation occurs differs from the air environment, although the main regularities remain.

The paper [9] deals with the sedimentation of mineral dust from the atmosphere in Japan. Satellite observations with remote sensing are used. The work has an ecological aspect. Dependences of dust sedimentation on time and space factors are investigated. The work uses satellite observations, and it can be considered as experimental. There are also training aids, for example, [8] which outlines the main provisions of theoretical calculations of the movement of dust or aerosols.

Indeed, all mathematical models combine the fact that in all we take into account gravity, the Archimedes' force and the viscous drag force of the medium, written as a rule in a differential form. However, the considered models do not consider the dust generated during abrasive cutting of stone structures consisting of a large number of minerals. In addition, the effects of the shape of particles and the state of the environment are almost not considered. Naturally, the issues of dust emission from under the cut circle, the concentration of dust in the working area and the issues of the cutting cycle duration are not considered.

Bearing in mind the considered materials in the introduction and in the review of the literature, the aim and objectives of this paper can be formulated as follows.

\section{The aim and objectives of research}

The aim of research is performing an analytical study of the change in the concentration of dust formed during the abrasive cutting of stone structures, depending on the rate of its sedimentation, which is a function of the size and shape of the dust, the material being cut, the temperature and the humidity of the environment.

To do this, it is necessary to solve the following tasks:

1. Develop a mathematical model of dust release from under the circle, and determine the distance to which it is ejected, depending on the rotation speed of the cutting circle.

2. Develop a mathematical model that adequately reflects the speed of sedimentation of dust particles depending on their material, the size and shape of these particles, and also depending on the state of the environment - temperature, pressure and humidity.

3. Using the obtained materials, as well as data from previous works published by the authors, which consider the intensity and productivity of dust generation during abrasive cutting of stone structures, calculate the dust concentration in the working space during the cutting cycle.

\section{Mathematical model of the dust release from under the circle. The general equation of motion}

In practical problems, as a rule, not individual particles are considered, but a cloud of particles of a dispersed phase, which includes hundreds and millions of individual particles. However, in the case of their low concentration, the motion of each particle does not depend on neighboring particles. And then, knowing the trajectory and velocities of individual particles, it is possible to calculate the behavior of the entire disperse system. The condition for the applicability of this approach is the low value of the volume concentration of particles.

The general vector equation of motion of a particle has the form $[2,3,9,10]$

$$
\mathrm{m}_{\mathrm{p}} \frac{\mathrm{d} \mathbf{v}}{\mathrm{dt}}=\mathbf{F}
$$




$$
\frac{\mathrm{d} \mathbf{R}_{\mathrm{p}}}{\mathrm{dt}}=\mathbf{v},
$$

where $\mathrm{m}_{\mathrm{p}}$ is the mass of a particle; $\mathbf{R}_{\mathrm{p}}$ is the radius vector of the particle coordinate; $\mathrm{F}$ is the vector of forces acting on the particle.

Forces acting on a particle (a speck of dust):

In the gravitational field, the gravitational force $\mathbf{F}_{\mathrm{g}}=\rho_{\mathrm{p}} \mathrm{V}_{\mathrm{p}} \mathbf{g}$ and the Archimedes force $\mathbf{F}_{\mathrm{A}}=$ $=-\rho \mathrm{V}_{\mathrm{p}} \mathbf{g}$ act on the particle (both resting and moving), which are written as the sum of the forces

$$
\mathbf{F}_{\mathrm{B}}=\left(\rho_{\mathrm{p}}-\rho\right) \mathrm{V}_{\mathrm{p}} \mathbf{g}
$$

where $V_{p}$ is the volume of a particle; $g$ is the acceleration vector of gravity; $\rho_{p}, \rho$ - the density of the particle material and the density of the dispersion medium, respectively.

Force of viscous resistance of medium

$$
\mathbf{F}_{\mathrm{S}}=-\mathrm{C}_{\mathrm{D}} \frac{\rho \mathrm{S}_{\mathrm{m}}}{2}\left|\mathbf{u}_{\mathrm{R}}\right| \mathbf{u}_{\mathrm{R}}
$$

where $\mathrm{S}_{\mathrm{m}}$ is the area of the median section of the body (the maximum cross-sectional area of the body in the plane perpendicular to the direction of its motion); $\mathbf{u}_{\mathrm{R}}=\mathbf{v}-\mathbf{u}$ is the velocity vector of the relative motion of the body and the carrier medium; the vectors $\mathrm{v}, \mathrm{u}$ are the velocities of the particle and the carrier medium, respectively; $C_{D}-$ empirical coefficient of resistance depending on the Reynolds number

$$
\operatorname{Re}=\frac{\rho\left|\mathbf{u}_{\mathrm{R}}\right| \mathrm{d}_{\mathrm{p}}}{\mu},
$$

$\mu$ - coefficient of the dynamic viscosity of the dispersion medium, if expressed through the coefficient of kinematic viscosity $v=\mu / \rho$, then the Reynolds number will take the form

$$
\operatorname{Re}=\frac{\left|\mathbf{u}_{\mathrm{R}}\right| \mathrm{d}_{\mathrm{p}}}{\mathrm{v}}
$$

$\mathrm{d}_{\mathrm{p}}$ is the particle diameter. Dependences of the $\mathrm{C}_{\mathrm{D}}$ values on the Reynolds number are presented in Table $1[9,10]$

Table 1

Dependences of $C_{D}$ values on the Reynolds number

\begin{tabular}{cc}
\hline Reynolds number, Re & $\mathbf{C}_{\mathbf{D}}$ \\
\hline $\operatorname{Re}<0.1$ & $24 / \operatorname{Re}$ \\
$0.1<\operatorname{Re}<2$ & $C_{D}=\frac{24}{\operatorname{Re}}\left[1+\frac{3}{16} \operatorname{Re}+\frac{9}{160} \operatorname{Re}^{2} \ln (2 \operatorname{Re})\right]$
\end{tabular}

When the particle is unsteady $\left(\mathrm{du}_{\mathrm{p}} / \mathrm{dt} \neq 0\right)$, an additional resistance force acts on it, due to the need to report acceleration to the motion of the mass of the carrier medium displaced by the particle

$$
\mathbf{F}_{\mathrm{R}}=-\frac{1}{2} \rho \mathrm{V}_{\mathrm{p}} \frac{\mathrm{d} \mathbf{v}}{\mathrm{dt}}
$$


This force, called the force of the attached masses, is directed opposite to the particle acceleration vector.

When the particle moves in a shear flow (with a non-uniform velocity profile of the carrier medium), the Saffman force (lift) acts on it, directed perpendicular to the particle motion vector. At low Reynolds numbers and in the absence of rotational motion of the particle, the Saffman force is given by Eq.

$$
\mathbf{F}_{\mathrm{S}}=\mathrm{C}_{\mathrm{S}} \mathrm{d}_{\mathrm{p}} \mathbf{u}_{\mathrm{R}} \sqrt{\rho \mu\left|\frac{\mathrm{d} \mathbf{u}}{\mathrm{dy}}\right|} \operatorname{sgn}\left(\frac{\mathrm{du}}{\mathrm{dy}}\right),
$$

where $\mathrm{C}_{\mathrm{S}}=1.615, \mathrm{du} / \mathrm{dy}$ is the transverse velocity gradient.

Substituting all the forces that are significant for the problem under consideration in equation (1), it is possible to obtain the differential equation of motion of the particle for each particular problem.

In the case of particles with a low inertia, the velocity of the dispersed phase is determined from the dynamic balance of forces acting on the particles. Thus, it is not necessary to solve the complete differential equations of motion, but it is sufficient to consider the equation of the dynamic balance of forces.

Let's start from the following assumptions:

- the motion of the particle is determined by the force of Archimedes $\mathbf{F}_{\mathrm{A}}$ and the force of resistance $\mathbf{F}_{\mathrm{S}}$;

- the particle velocity vector $\mathbf{v}$ at the initial instant of time is parallel to the acceleration vector caused by the Archimedes force $\mathbf{F}_{\mathrm{A}}$;

- there is no interaction between the particles;

Taking into account the above assumptions, the equation of motion of a single particle can be represented in the following form:

$$
\mathrm{m} \frac{\mathrm{d} \mathbf{v}}{\mathrm{dt}}=-\mathrm{C}_{\mathrm{D}} \frac{\rho \mathrm{S}_{\mathrm{m}}}{2}|\mathbf{v}| \mathbf{v}+\left(\rho_{\mathrm{p}}-\rho\right) \mathrm{V}_{\mathrm{p}} \mathbf{g}
$$

Let's build the trajectories of dust particles on the basis of the Runge-Kutta integration of the equations of motion (1), which for the two-dimensional case, taking into account that the velocity of the medium is zero, will be written as follows

$$
\begin{gathered}
\frac{d v_{i}}{d t}=-C_{D} \frac{\rho S_{m}}{2 m_{p}}|v| v_{i}-\frac{\left(\rho_{p}-\rho\right)}{m_{p}} v_{p} g_{i} \delta_{i 2},\left|v_{i}\right|=\sqrt{v_{1}^{2}+v_{2}^{2}}, \\
\frac{d x_{i}(t)}{d t}=v_{i}(t),
\end{gathered}
$$

where $\mathrm{x}_{\mathrm{i}}(\mathrm{t})$ are the coordinates of the particle position; $\mathrm{v}_{\mathrm{i}}(\mathrm{t})$ is the velocity of the particle; $\delta-$ the symbol of Kronecker.

With the initial conditions $\mathrm{x}_{\mathrm{i}}(0)=0, \mathrm{i}=1,2,\left.\quad\left\{\mathrm{v}_{1}(\mathrm{t}), \mathrm{v}_{2}(\mathrm{t})\right\}\right|_{\mathrm{t}=0}=\left\{\mathrm{v}_{1}^{0}, \mathrm{v}_{2}^{0}\right\}$.

In the case of a spherical particle, the system (1.3) will be written as

$$
\begin{gathered}
\frac{d v_{i}}{d t}=-C_{D} \frac{3}{4 d_{p}}|v| v_{i}-\frac{\left(\rho_{p}-\rho\right)}{\rho_{p}} g_{i} \delta_{i 2},|v|=\sqrt{v_{1}^{2}+v_{2}^{2}}, \\
\frac{d x_{i}(t)}{d t}=v_{i}(t) .
\end{gathered}
$$

Numerical studies of the dependence of the velocity of particles and the height on which they rise as a function of the geometrical parameters of the particle are shown in Fig. 1. 
The present and subsequent calculations are carried out for conditions: atmospheric pressure $100 \mathrm{kPa}$, air humidity $60-80 \%$. Under these conditions, work is usually done and fluctuations in air density are insignificant.

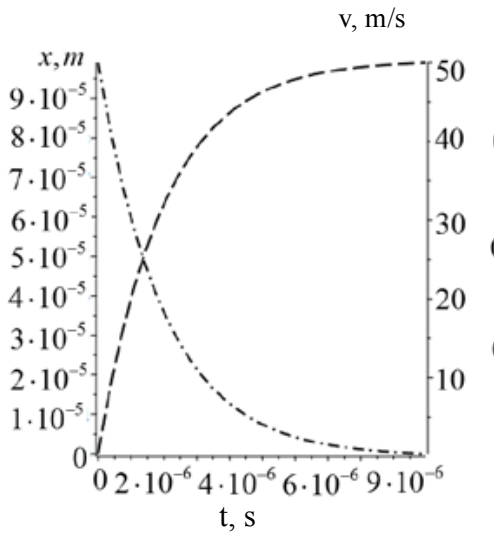

$a$

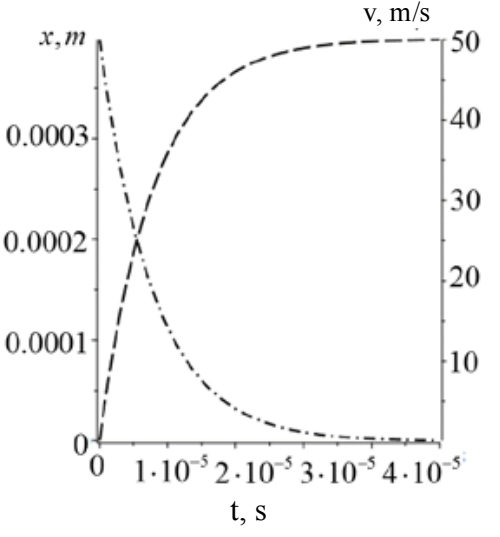

$b$
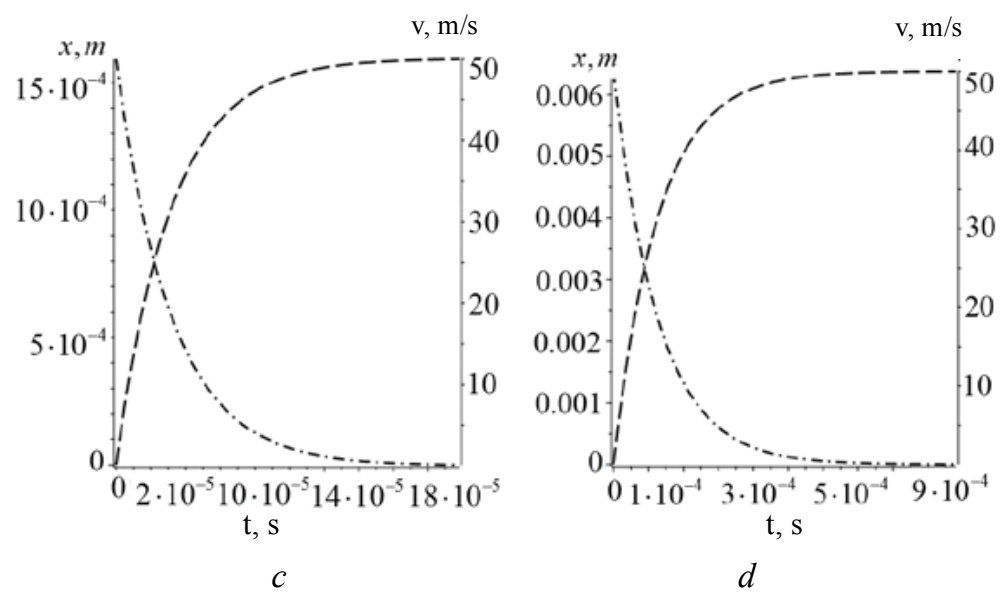

Fig. 1. Dependence of particle velocity and lift height as a function of time and particle diameter $\mathrm{d}_{\mathrm{p}}$. The particle velocity is a dash-dot line; the height of the emission of particles is a dashed line.

The material is basalt, $a-\mathrm{d}_{\mathrm{p}}=0.5 \mu \mathrm{m}, b-\mathrm{d}_{\mathrm{p}}=1 \mu \mathrm{m}, c-\mathrm{d}_{\mathrm{p}}=2 \mu \mathrm{m}, d-\mathrm{d}_{\mathrm{p}}=4 \mu \mathrm{m}$

From Fig. 1 it can be seen that at a certain moment of time the velocity of the particle will drop to zero and the particle becomes to a so-called suspended state.

Velocity and sedimentation time of particles.

The basic condition necessary for finding a particle in the suspended state, and, consequently, determining the sedimentation of particles is the condition for the dynamic balance of forces (2), which in the case of gravitational sedimentation of particles, ie, in the stationary mode $(\mathrm{dv} / \mathrm{dt}=0)$, can be represented as follows

$$
-\mathrm{C}_{\mathrm{D}} \frac{\rho \mathrm{S}_{\mathrm{m}}}{2 \mathrm{~m}}\left|\mathrm{v}_{\mathrm{s}}\right| \mathrm{v}_{\mathrm{s}}+\frac{\rho_{\mathrm{p}}-\rho}{\mathrm{m}} \mathrm{V}_{\mathrm{p}} \mathrm{g}=0
$$

where $v_{s}$ - the rate of steady-state sedimentation of the particle (sedimentation rate).

Using the solution of equation (5), it is possible to determine such characteristics as the sedimentation time $t_{\mathrm{s}}$ and the sedimentation length $1_{\mathrm{s}}$ as follows:

$$
\begin{gathered}
t_{s}=\frac{\rho}{\rho-\rho_{p}} \frac{v_{s}}{g}, \\
1_{s}=v_{s} t_{s} .
\end{gathered}
$$


The sedimentation rate can be considered as a scale characteristic characterizing the velocity of the particle in a resisting medium.

\section{Influence of the particle shape on the sedimentation rate.}

The shape of the particles of the dispersed phase can differ from the spherical phase (snowflakes, polyhedra, ellipsoids, plates, fibers, etc.), Fig. 2.

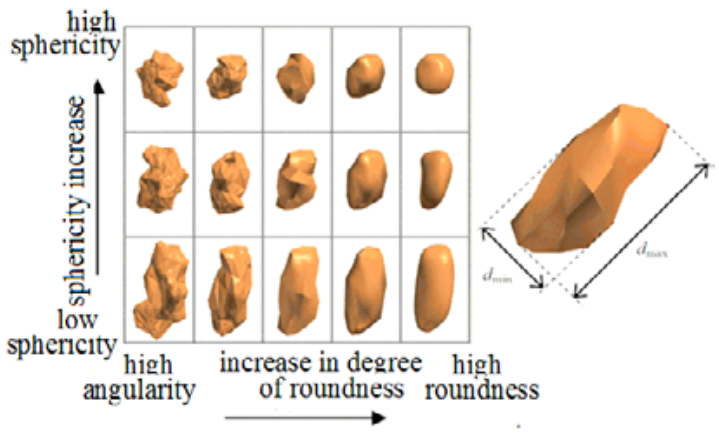

Fig. 2. Forms of dispersed phase particles

Since the methods of dispersion analysis do not, in their majority, allow fully characterize each particle of a disperse system in three dimensions, let's use an approximation, in other words, the replacement of particles of real material by equivalent particles of regular geometric shape. When analyzing a particle under a microscope, its planar projection is visualized, in which case the particle can be characterized by a number of different dimensional parameters. It is important to understand that each method of determining the size is based on measuring the various physical characteristics of particles (maximum length, minimum length, volume, surface area etc.), and as a result, the sizes obtained by different methods will differ. Fig. 3 shows the various options for answering the question what is the particle size. At the same time there are no erroneous results each answer is subjectively correct - it reflects a physically measured characteristic.

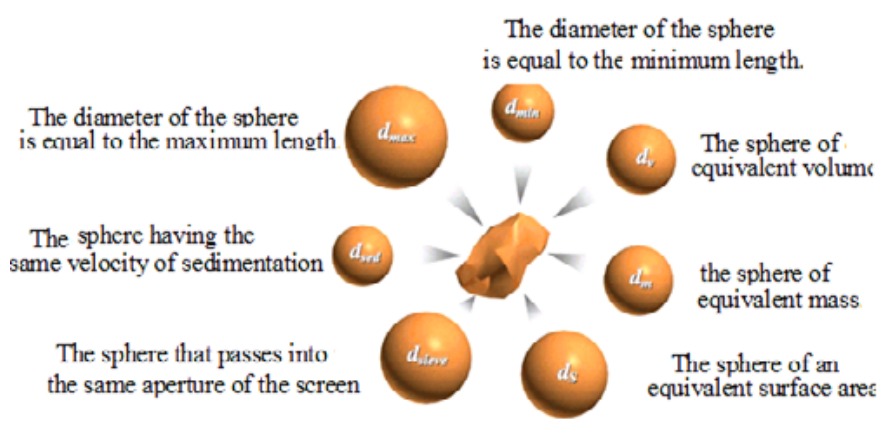

Fig. 3. Equivalent diameters of dispersed phase particles

When calculating the motion of non-spherical particles in the corresponding equations instead of $d_{p} d_{p e}$ is substituted. It should be noted that in the case of flow past particles whose shape differs from spherical, this approach gives only an estimated result. For clarification, it is necessary to carry out experiments with a spherical and predetermined particle (for example, gravitational sedimentation in a viscous liquid) and introduce a correction factor (geometric coefficient).

For non-spherical particles, a formula for $C_{D}$ was proposed in [10] for, taking into account the sphericity factor $\Phi_{\mathrm{p}}$

$$
\mathrm{C}_{\mathrm{D}}=\frac{24}{\operatorname{Re}}\left[1+\frac{3}{16} \mathrm{Re}+\frac{9}{160} \operatorname{Re}^{2} \ln (2 \mathrm{Re})+\frac{10\left(1-\Phi_{\mathrm{p}}\right)}{\Phi_{\mathrm{p}}} \mathrm{Re}^{0.35}\right], \quad \mathrm{Re}<2,
$$

where $\Phi_{\mathrm{p}}=\pi\left(6 \mathrm{~V}_{\mathrm{p}} / \pi\right)^{2 / 3} \mathrm{~S}_{\mathrm{p}}^{-1} ; \mathrm{V}_{\mathrm{p}}-$ the equivalent volume of a particle. 
For particles of complex shape, the difficulties that arise when the dynamic and geometric parameters of the particles are compared can be overcome by replacing real particles with ellipsoids close in shape.

Let's consider a particle in the form of an elongated ellipsoid:

$$
\begin{gathered}
\Omega: x^{2} / a^{2}+y^{2} / b^{2}+z^{2} / b^{2}=1, a>b \\
V_{p}=4 \pi a b^{2} / 3 \\
S_{p}=2 \pi b^{2}\left(1+\lambda e^{-1} \arcsin (e)\right) \\
\lambda=a / b>1 \\
e=a^{-1} \sqrt{a^{2}-b^{2}}
\end{gathered}
$$

where $\mathrm{V}_{\mathrm{p}}$ is the volume of the particle, and $\mathrm{S}_{\mathrm{p}}$ is the surface area of the particle.

Then, the sphericity factor $\Phi_{p}$ is written as follows:

$$
\Phi_{\mathrm{p}}=\Phi_{\mathrm{p}}(\lambda)=2 \lambda^{2 / 3}\left(1+\lambda^{2}\left(\lambda^{2}-1\right)^{-1 / 2} \arcsin \sqrt{1-\lambda^{-2}}\right)^{-1} .
$$

The projected area $A_{p}$ will depend on the orientation of the main axis of the ellipsoid relative to the vertical. The orientation will depend on the initial conditions, particle inertia and environmental conditions. If consider a rotation $\theta$ in the $(\mathrm{x}, \mathrm{y})$ plane, it is an ellipse in the projection. For randomly oriented particles, the projected area $A_{p}$ is replaced by a spherically averaged value

$$
\hat{\mathrm{A}}_{\mathrm{p}}=\left[4 \int_{0}^{\pi / 2} \pi \mathrm{ba}_{\text {proj }}(\theta) \mathrm{d} \theta\right]\left[4 \int_{0}^{\pi / 2} \mathrm{~d} \theta\right]^{-1}=2 \mathrm{baE}(\mathrm{e})
$$

where $\mathrm{E}(\mathrm{e})$ is a complete elliptic integral of the second kind.

Finally, let's obtain an expression for the equivalent diameter $d_{p e}$ as a function of $\lambda$

$$
d_{p e}=d_{p e}(\lambda)=2\left(S_{p} / \pi\right)^{1 / 2}=2 b\left(1+\frac{\lambda^{2}}{\left(\lambda^{2}-1\right)^{1 / 2}} \arcsin \sqrt{1-\lambda^{-2}}\right)^{1 / 2}=b \Psi_{d}(\lambda) .
$$

Substituting the obtained expressions into the equation of the balance of forces (7), let's obtain

$$
\begin{gathered}
\mathrm{v}_{\mathrm{s}}=\frac{\left(\rho_{\mathrm{p}}-\rho\right)}{12} \frac{\mathrm{d}_{\mathrm{p}} \mathrm{V}_{\mathrm{p}} \mathrm{g}}{\mu \mathrm{S}_{\mathrm{m}}}=\frac{\left(\rho_{\mathrm{p}}-\rho\right)}{18 \mu} \frac{\pi \mathrm{d}_{\mathrm{p}}^{2}}{\Psi_{\mathrm{d}}(\lambda) \mathrm{E}(\mathrm{e})}, \quad \mathrm{Re}<1, \\
{\left[1+\frac{3}{16}\left(\frac{\rho \mathrm{v}_{\mathrm{s}} \mathrm{d}_{\mathrm{p}}(\lambda)}{\mu}\right)+\frac{9}{160}\left(\left(\frac{\rho \mathrm{v}_{\mathrm{s}} \mathrm{d}_{\mathrm{p}}(\lambda)}{\mu}\right)\right)^{2} \ln \left(\frac{2 \rho \mathrm{v}_{\mathrm{s}} \mathrm{d}_{\mathrm{p}}(\lambda)}{\mu}\right)+\right.} \\
\left.+\frac{10\left(1-\Phi_{\mathrm{p}}(\lambda)\right)}{\Phi_{\mathrm{p}}(\lambda)}\left(\frac{\rho \mathrm{v}_{\mathrm{s}} \mathrm{d}_{\mathrm{p}}(\lambda)}{\mu}\right)^{0.35}\right] \mathrm{v}_{\mathrm{s}}=\frac{\left(\rho_{\mathrm{p}}-\rho\right)}{18 \mu} \frac{\mathrm{d}_{\mathrm{p}}^{2}(\lambda) \pi \mathrm{g}}{\mathrm{E}(\mathrm{e}) \Psi_{\mathrm{d}}(\lambda)}, \quad 1<\operatorname{Re}<2 .
\end{gathered}
$$

Equation (15) is nonlinear with respect to $\mathrm{v}_{\mathrm{s}}$ and must be solved numerically.

In Fig. 4 is shown the dependence of the particle sedimentation rate $(\mathrm{cm} / \mathrm{h})$ on its geometric parameters and temperature at atmospheric pressure for the case $\operatorname{Re}<1$.

In Fig. 5 is shown the dependence of the sedimentation rate as a function of the material and the geometric parameters of the particle at atmospheric pressure for the $\mathrm{Re}<1$ case. 


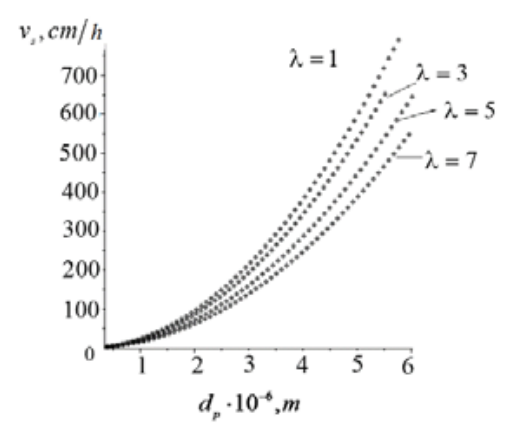

$a$

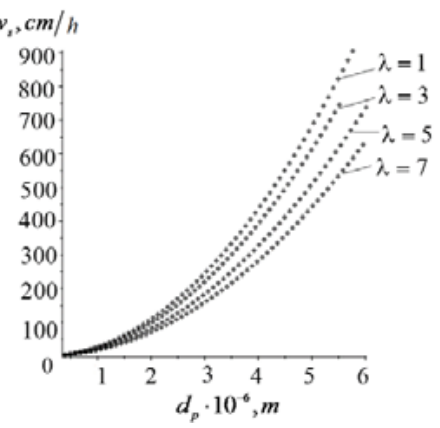

b

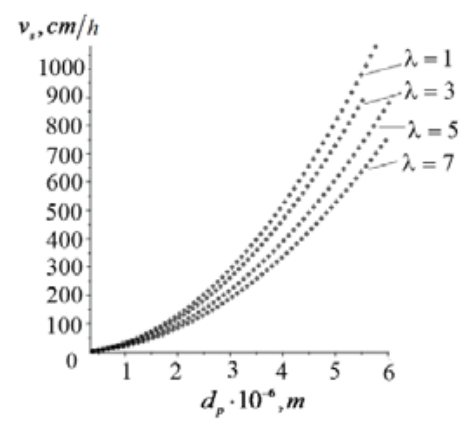

$c$

Fig. 4. Dependence of the sedimentation rate $(\mathrm{cm} / \mathrm{h})$ on the geometric parameters of the particle and the ambient temperature. At $\lambda=1$ (spherical particle), diameters $d_{p}=0.4 \ldots 6 \mu \mathrm{m}$, when taken, $\lambda=3.5 .7$, the value of $\mathrm{b}$ was chosen so that $\mathrm{d}_{\mathrm{pe}}=\mathrm{d}_{\mathrm{pe}}(\lambda)=0.4 .6 \mu \mathrm{m}$. Cut material - basalt, $a-\mathrm{T}=20{ }^{\circ} \mathrm{C}$, $b-\mathrm{T}=40^{\circ} \mathrm{C}, c-\mathrm{T}=-20^{\circ} \mathrm{C}$

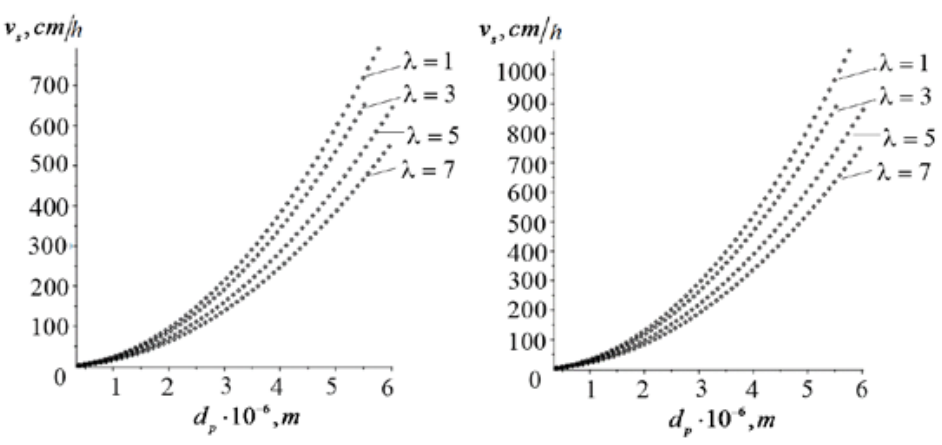

a

$b$

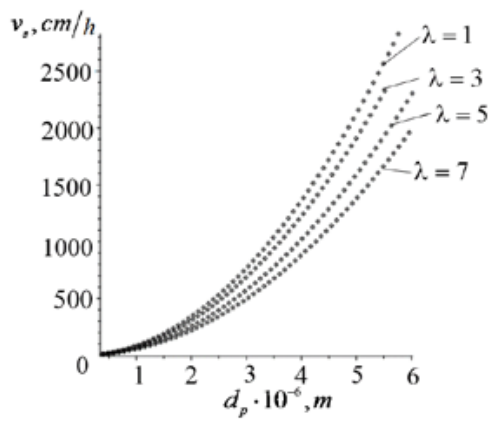

$c$

Fig. 5. Dependence of sedimentation rate $(\mathrm{cm} / \mathrm{h})$ on the type of material being cut and the geometrical parameters of the particle: $a$ - concrete $\left(\rho_{\mathrm{p}}=2200 \mathrm{~kg} / \mathrm{m}^{3}\right)$,

$b$-granite $\left(\rho_{\mathrm{p}}=3000 \mathrm{~kg} / \mathrm{m}^{3}\right), c-$ steel $40 \mathrm{X}$ (carbon $0.4 \%$, chromium $1 \%, \rho_{\mathrm{p}}=7850 \mathrm{~kg} / \mathrm{m}^{3}$ ) 


\section{Model for determining the concentration of particles}

The concentration of dispersed particles is also one of the most important characteristics on which the behavior of the disperse system depends on various physicochemical processes. There are countable $\mathrm{C}_{\mathrm{n}}$, volumetric $\mathrm{C}_{\mathrm{V}}$ and mass $\mathrm{C}_{\mathrm{m}}$ concentration of particles:

$\mathrm{C}_{\mathrm{n}}$ is the number of particles per unit volume of a two-phase medium;

$\mathrm{C}_{\mathrm{m}}$ is the total mass of particles per unit volume of a two-phase medium;

$\mathrm{C}_{\mathrm{v}}$ is the total volume of particles per unit volume of a two-phase medium.

The relationships between $\mathrm{C}_{\mathrm{n}}, \mathrm{C}_{\mathrm{m}}, \mathrm{C}_{\mathrm{v}}$ for polydisperse systems (consisting of particles of different sizes) are presented in (Table 2) [8]. For such systems, the dispersed composition is characterized by a differential function of the countable particle size distribution $f(D)=(a / D) \cdot \exp \left(-b \ln (\beta D)^{2}\right)$.

Table 2

Relationships between concentrations for polydisperse media [8]

\begin{tabular}{|c|c|c|c|}
\hline & $\mathrm{C}_{\mathrm{n}}$ & $\mathrm{C}_{\mathrm{m}}$ & $\mathrm{C}_{\mathrm{V}}$ \\
\hline $\mathrm{C}_{\mathrm{n}}$ & $C_{n}$ & $C_{m}\left[\frac{\pi \rho_{p}}{6} \int_{0} D^{3} f(D) d D\right.$ & $C_{v}\left[\frac{\pi}{6} \int_{0} D^{3} f(D) d D\right]$ \\
\hline $\mathrm{C}_{\mathrm{m}}$ & $C_{n} \frac{\pi \rho_{p}}{6} \int_{0}^{\infty} D^{3} f(D) d D$ & $\mathrm{C}_{\mathrm{m}}$ & $\mathrm{C}_{\mathrm{v}} \rho_{\mathrm{p}}$ \\
\hline $\mathrm{C}_{\mathrm{v}}$ & $C_{n} \frac{\pi}{6} \int_{0}^{\infty} D^{3} f(D) d D$ & $\mathrm{C}_{\mathrm{m}} \frac{1}{\rho_{\mathrm{p}}}$ & $\mathrm{C}_{\mathrm{v}}$ \\
\hline
\end{tabular}

Let's consider the countable concentration of dust $\mathrm{C}_{\mathrm{n}} \equiv \mathrm{C}(\mathrm{y}, \mathrm{t})$, for the calculation of which, using gravitational sedimentation, we use the following differential equation, which is a two-dimensional transport equation

$$
\begin{gathered}
\frac{\partial \mathrm{C}_{\mathrm{i}}(\mathrm{y}, \mathrm{t})}{\partial \mathrm{t}}-\mathrm{u}_{\mathrm{s}, \mathrm{i}} \frac{\partial \mathrm{C}_{\mathrm{i}}(\mathrm{y}, \mathrm{t})}{\partial \mathrm{y}}=\mathrm{Q}_{\mathrm{i}}(\mathrm{t}) \delta\left(\mathrm{y}-\mathrm{y}_{\mathrm{i}}\right) \\
\left.\mathrm{C}_{\mathrm{i}}(\mathrm{y}, \mathrm{t})\right|_{\mathrm{t}=0}=0,\left.\mathrm{C}_{\mathrm{i}}(\mathrm{y}, \mathrm{t})\right|_{\mathrm{y}=\mathrm{y}_{\mathrm{i}}}=0
\end{gathered}
$$

where $\mathrm{C}_{\mathrm{i}}(\mathrm{y}, \mathrm{t}) \mathrm{u}_{\mathrm{s}, \mathrm{i}}$, respectively, the concentration and velocity of the i-th fraction of the dust; $\mathrm{Q}_{\mathrm{i}}(\mathrm{t})=\mathrm{Q} \sum_{\mathrm{j}=1}^{60} \eta(\mathrm{t}-\mathrm{j})$ - intensity of dusting; $\eta(\mathrm{t}-\tau)$ is the Heaviside function; $\delta\left(\mathrm{y}-\mathrm{y}_{\mathrm{i}}\right)$ is the Dirac delta function by which the height of the i-th fraction of dust is taken into account.

In order to avoid the application of the Laplace transform and difficulties with its treatment, we proceed as follows. Let's divide the time interval $\mathrm{T}$ into $\mathrm{M}$-intervals of length $\mathrm{h}=\mathrm{TM}^{-1}$ and in formula (16) replace the derivative with respect to time by the difference relation:

$$
\frac{\partial \zeta}{\partial t}=\frac{\zeta_{j}-\zeta_{j-1}}{h}, \zeta_{j}(y)=\zeta(y, j h), j=1,2, \ldots
$$

As a result,

$$
\frac{\partial C_{i, j}(y)}{\partial y}-\frac{1}{u_{s, i} h} C_{i, j}(y)=\frac{1}{u_{s, i}} Q_{i, j} \delta\left(y-y_{i}\right)-\frac{1}{u_{s, i} h} C_{j-1}(y), \quad j=1 . . N,
$$




$$
\mathrm{C}_{\mathrm{i}, 0}(\mathrm{y})=0,\left.\mathrm{C}_{\mathrm{i}, \mathrm{j}}(\mathrm{y})\right|_{\mathrm{y}=\mathrm{y}_{\mathrm{i}}}=0
$$

Suppose $j=1$, the equation takes the form

$$
\frac{\partial \mathrm{C}_{\mathrm{i}, 1}(\mathrm{y})}{\partial \mathrm{y}}-\frac{1}{\mathrm{u}_{\mathrm{s}, \mathrm{i}} \mathrm{h}} \mathrm{C}_{\mathrm{i}, 1}(\mathrm{y})=\frac{1}{\mathrm{u}_{\mathrm{s}, \mathrm{i}}} \mathrm{Q}_{\mathrm{i}, 1} \delta\left(\mathrm{y}-\mathrm{y}_{\mathrm{i}}\right), \quad \mathrm{j}=1 . . \mathrm{N} .
$$

With allowance for the initial conditions, the solution of equation (18) has the form

$$
C_{1}(y)=Q_{i, 1}\left[\eta\left(y-y_{i}\right)-1 / 2\right] e^{\frac{y-y_{i}}{h \cdot u_{s, i}}}
$$

substituting it in (17) and continuing the iterative process, let's obtain the values of the concentration of the $\mathrm{i}$-th dust fraction over time.

At each model time $\Delta \mathrm{t}=1$ s. $4.6 \cdot 10^{5}-2.8 \cdot 10^{6}$ particles of different diameters arrive in the work zone [1]. Using (15), it is found that the minute concentration of dust during the cutting of a stone (in the dimensions of the working zone $0.5 \cdot 0.5 \cdot 0.5 \mathrm{~m}=0.125 \mathrm{~m}^{3}$ ) is in the range $0.28 \cdot 10^{8}-$ $1.68 \cdot 10^{8} \mathrm{pcs} / \mathrm{m}^{3}$, for the cutting time of $3 \mathrm{~m}$ let's obtain a concentration of $0.84 \cdot 10^{8}-4.8 \cdot 10^{8} \mathrm{pcs} / \mathrm{m}^{3}$. The range of sizes of dust particles is $0.35-6 \mu \mathrm{m}$.

\section{Discussion of the results of the analytical study}

The results of the calculations show that the sedimentation velocity of particles depends significantly on the shape. The higher the sphericity, the higher the rate of sedimentation. Obviously, irregularly shaped particles experience greater medium resistance and greater Archimedean forces, which somewhat compensates for the gravity force under which the dust particle "falls". The temperature state of the environment has little effect on the sedimentation rate, in any case in the accepted temperature range at which work can occur. This can be explained by a slight change in the density of air, as a result of which the force of the medium's resistance and the Archimedean force vary insignificantly.

The sedimentation rate of dust particles generated by cutting the most common building stone materials also differs slightly. So practically the same sedimentation rate has dust particles obtained by cutting basalt and concrete. A bit higher is the rate of sedimentation of particles from granite. This can be explained by the fact that the structure of these materials is fragile, as a result of which the dust particles have a similar shape and the sedimentation rate is affected only by the density, which differs insignificantly. As for particles of chips - dust when cutting steel reinforcement, the influence of a higher density of steel is clearly visible here. In addition, the chips formed as a result of plastic deformation are likely to have a more "streamlined" shape and experience less air resistance. This circumstance, however, requires additional study.

The sedimentation rate of particles of generated dust, taking into account the actual shape for shearing strain $(\lambda=5-7)$, is approximately $600-700 \mathrm{~cm} / \mathrm{h}$ or $10-11 \mathrm{~cm} / \mathrm{min}$ for particles measuring $6 \mu \mathrm{m}$. This means that at a production height of about $2 \mathrm{~m}(200 \mathrm{~cm})$ during the operating cycle (about $3 \mathrm{~min}$ ), the dust will remain at an altitude of about $1.5 \mathrm{~m}$, i. e. practically remains in the working area. This gives grounds to assert about a high concentration of dust (as indicated by the order of $4.8 .10^{8} \mathrm{pcs} / \mathrm{m}^{3}$ ). It should be noted that particles of $4-6 \mu \mathrm{m}$ in size were taken into account. Taking into account smaller particles, this concentration will be somewhat higher.

The advantage of the analytical study can be considered that mathematical models make it possible to evaluate the generation of dust when cutting various materials, for example, ceramic tiles from aluminum oxides and zirconium.

It should be noted that the study does not exhaust the topic of dust generation in the abrasive cutting of stone materials. Further research should be directed to the investigation of air currents arising during the rotation of the circle, the interaction of these flows with dust and with cooling liquids. 


\section{Conclusions}

1. A mathematical model of dust extraction from under the circle is developed, the distance to which it is ejected is determined, depending on the rotation speed of the cutting circle, and is on the order of 7-10 $\mathrm{mm}$ with a particle size of 4-6 $\mu \mathrm{m}$, with a circle rotation speed of about $35-50 \mathrm{~m} / \mathrm{s}$.

2. A mathematical model has been developed that adequately reflects the sedimentation velocity of dust particles depending on their material, size and shape, and also depending on the state of the environment. It is shown that the sedimentation velocity of dust particles with dimensions of $4-6 \mu \mathrm{m}$ is $10-11 \mathrm{~cm} / \mathrm{min}$. The more a dust particle differs from a spherical shape, the lower its sedimentation rate. The state of the environment under the conditions under which the work is performed has little effect on the rate of dust sedimentation. The type of cutting stone material basalt, granite, concrete - has little effect on the sedimentation velocity, since the densities of these materials are quite close. Dimensions of dust particles significantly affect the sedimentation rate. So when the size of dust particles decreases from 6 to $2 \mu \mathrm{m}$, i.e. 3 times, the sedimentation rate decreases by 7 times.

3. The conducted research allows to determine the dust concentration in the working space in the amount of $4.8 \cdot 10^{8} \mathrm{pcs} / \mathrm{m}^{3}$ during the working cycle of cutting, that allows in each case to formulate the requirements shown to means of individual and collective protection of workers.

\section{References}

[1] Bezpalova, A., Lebedev, V. (2017). Investigation of the formation process of hazardous and harmful production factors when cutting a stone for construction works. EUREKA: Physics and Engineering, 5, 30-38. doi: 10.21303/2461-4262.2017.00416

[2] Gao, R., Li, A. (2011). Modeling deposition of particles in vertical square ventilation duct flows. Building and Environment, 46 (1), 245-252. doi: 10.1016/j.buildenv.2010.07.020

[3] Maggi, F. (2013). The settling velocity of mineral, biomineral, and biological particles and aggregates in water. Journal of Geophysical Research: Oceans, 118 (4), 2118-2132. doi: 10.1002/jgrc.20086

[4] Dust and respiratory tract. All about spontaneous pneumothorax. Available at: http://www.spontan.ru/pnevmokoniozy/186-pyl-i-dyxatelnye-p.uti.html

[5] Mushchenko, B. L. (2009). Calculation of the rate of incidence of dust particles and the evaluation of the degree of influence of various forces acting on the particle. Scientific Bulletin of the Voronezh State University of Architecture and Civil Engineering, 2, 58-63.

[6] Azarov, V. N., Bessarab, O. I., Kabaev, O. V. (2010). Theoretical studies of sedimentation rate of fine dust in the air of working rooms of engineering and construction industry enterprises. Bulletin of VolgGASU. Series: Str-in and the architect, 17 (36), 102-105.

[7] Osada, K., Ura, S., Kagawa, M., Mikami, M., Tanaka, T. Y., Matoba, S. et. al. (2014). Wet and dry deposition of mineral dust particles in Japan: factors related to temporal variation and spatial distribution. Atmospheric Chemistry and Physics, 14 (2), 1107-1121. doi: 10.5194/acp-14-1107-2014

[8] Arkhipov, V. A., Usanina, A. S. (2013). A877 Movement of aerosol particles in a stream. Tomsk: Publishing House of Tomsk State University, 92.

[9] Clift, R., Grace, J. R., Weber, M. E. (1978). Bubbles, Drops, and Particles. Academic Press, 380.

[10] Gu, Z., Zhao, Y., Li, Y., Yu, Y., Feng, X. (2006). Numerical Simulation of Dust Lifting within Dust Devils-Simulation of an Intense Vortex. Journal of the Atmospheric Sciences, 63 (10), 2630-2641. doi: $10.1175 /$ jas 3748.1 